\title{
Real-Time Intelligent Thermal Comfort Prediction Model
}

\author{
Farid Ali Mousa ${ }^{1}$ \\ Information Technology Department \\ Faculty of Computers and Artificial Intelligence \\ Beni-Suef University, Beni Suef, Egypt
}

\author{
Heba Hamdy $\mathrm{Ali}^{2}$ \\ Multimedia Department \\ Faculty of Computers and Artificial Intelligence \\ Beni-Suef University, Beni Suef, Egypt
}

\begin{abstract}
Real-time prediction model of indoor thermal comfort depending on Momentum Back Propagation (MBP) function is established by using Arduino hardware and mobile application. The air temperature indoor, air velocity, and relative humidity are gathered via temperature sensor and transferred via Bluetooth to the mobile application to predicate thermal comfort. A significant challenge in designing MBP is to decide the best architecture and parameters as the number of layers and nodes, and number of epochs for the network given the data for the AI issues. These parameters are usually selected on heuristic and fine-tuned manually, which could be as boring as the performance assessment may take hours to test the output of a single MBP parameterization. This paper tends to the issue of determining appropriate parameters for the MBP by applying chicken swarm optimization (CSO) algorithm. The CSO algorithm simulates the chicken swarm searching for the best parameter employs the Fitness function of these parameters which yielding minimum error and high accuracy. The proposed accuracy approximately equals $98.3 \%$ when using the best parameters obtained from Chicken Swarm Optimization (CSO). The proposed methodology performance is assessed on the collected dataset from weather archive and in the context of thermal comfort prediction, that mapping relations between the indoor features and thermal index.
\end{abstract}

Keywords-Thermal comfort; chicken swarm optimization; momentum back propagation; neural network; bio-inspired optimization algorithm

\section{INTRODUCTION}

In the production of building architecture, thermal comfort proves to be one of the most critical factors. People always wanted to create a thermal climate. Thermal comfort means a "condition of mind" in compliance with ISO 7730(1993) and ASHARE Standard 55(2010) [1, 2], which reflects pleasure with the thermal environment in which the thermal environment is situated'. Thus, in work, people must be pleased with the thermal atmosphere surrounding them or they will suffer from and won't work like they used to be. The principal necessity is preserving thermal comfort and it need to be achieved with the appropriate thermal equilibrium of the human nature. The basic thermal comfort factors can influence the safety or the health of the employees [3], e.g., the possibility of a rise in heat, decline or much worse could be if temperatures are too high. Also, the possibility of employee headaches, loss of focus or nausea, may also occur when the temperature drops too much. Being sleepy or not feel at all well when they function, all these could be happened due to the temperature in their offices or spaces. There are four fundamental thermal comfort factors [4]; first, moisture that ensures a significant amount of water is present in the air which keeps the sweat evaporation from the skin. Second, the ambient air temperature surrounding the body. Third, the air speed or the rapid flow of air in the employee's atmosphere that is the primary thermal comfort component. Thus, the air in a warmed indoor atmosphere will relax workers. There is a certain range of thermal comfort for each of the fundamental factors.

\section{RELATED WORK}

Thermal comfort is largely attributed to environmental and human influences. The fundamental adaptive thermal comfort theory stated that, people living in one place already seemed to be adapted to the thermal local environment [5] and their thermal history could contribute to different conditions of thermal comfort [6][7]. Thermal conditions in thermal systems can be more effectively modeled locally and internationally, helps to design and improve building thermal systems [8][9]. Thermal conditions in humans are best viewed in terms of their thermal comfort needs.

Two distinct models, named the adaptive model and the PMV/PPD model [10], can be calibrated according to literature for the thermal comfortability measurement. The predict mean vote (PMV) should be the conventional dominant thermal comfort model [11], based on the thermal equilibrium between the human body and the environment. The model based on the heat balance concepts and the data was obtained from the chamber experiment where detailed monitoring of indoor conditions could be accomplished. In terms of the four environmental and the two personal factors mentioned before, the PMV model presents a statistical model to forecast the thermal sensation of a wide group of subjects.

These six major thermal comfort factors are grouped into environmental factors are; air temperature, mean radiant temperature, relative humidity and air speed and personal factors are; metabolic rate and clothing insulation, that directly affect the thermal comfort [12]. The PMV/PPD model is appropriate with air conditioning buildings and ventilation systems, while the adaptive model is most suited with naturally conditioned buildings without mechanical condition systems [13].

ASHRAE uses the PMV index to estimate the average reaction of a broad seven-points thermal scale of a large 
number of people from cold (3) to hot (3) [14] this is known as the 'ASHRAE scale'. Zero stands for the desired value of thermal neutrality. A consumer will define a value similar to 0 for the PMV in a setting he/she finds convenient. The expected unmet percentage (PPD) is an indicator used to measure the proportion of people unfulfilled with a certain thermal condition feeling that is whether too cold or too hot as recommended from their PMV values [15].

The PPD index is thus closely connected to PMV. This dependency is seen in the Fanger equation [12].

The ThermCont model, that learns a regressor which takes the six vector parameters of PMV as an input and provides a corresponding PMV value as an output, is designed to avoid and monitor the thermal comfort of the occupant by using machine learning tools. ThermCont utilizes Multiple Linear Regression [16] (MLR) algorithm, which is focused on subjective thermal comfort findings performed in a building of an office [17]. In addition, a genetic algorithm [18] has been developed to detect thermal comfort (PMV) in real time to enhance thermal comfort for indoor people.

Data-driven approach [19] is developed to forecast thermal comfort of individual in real-time using a range of human factors and environmental factors including the six Fanger elements and the three new factors which are; gender, age and outdoor weather. The outdoor weather and three new features were added. Eventually, data of the outdoor weather was used by effective temperature because effective temperature is reflective of the weather, unlike air temperature.

The ANN is practically utilized to estimate non-linear relationships between input features and output [20]. The artificial neural networks are applied to predict PMV index values of thermal comfort in a room. In order to select the right conditions, the ANN modeling can be performed many times. But the globally optimal solution in this case is not guaranteed. We propose in this paper a better way to address this issue by using CSO to ensure an optimum modeling parameter for a neural network is the Global Minimum Square Error (MSE). The use of the CSO means that the minimum number of time slots generated also matches the real-time limit.

This paper is organized as follows: in Section 3, the overall architecture of the proposed real-time intelligent thermal comfort prediction model is introduced. In Section 4, the proposed methodology that solves the predication problem is explained. Section 5 introduces and discusses the experimental and finally the conclusion in Section 6.

\section{REAL-TIME INTELLIGENT THERMAL COMFORT PREDICTION MODEL}

Fig. 1 shows the indoor air speed, air temperature and relative humidity are gathered by hardware interface. The Hardware interfaces includes the components; LM35 temperature sensor, Ardunio-uno and Bluetooth HC-05. The data are collected using the temperature sensor (LM5) which transferred using Ardunio-uno hardware. Then the data are transmitted via hardware to connected Bluetooth. The Bluetooth send the collected data to the mobile application for thermal comfort prediction. To receive the last temperature, form the indoor atmosphere, the user just presses a button in the application. Since the data was received in Fahrenheit, it has to be converted into Celsius. The gathered data are sent via cellular network or $\mathrm{WiFi}$ to trained model to predict thermal comfort which is then sent back to the mobile application according to Table I.

\section{A. Data Collection from Arduino}

The current air speed, relative humidity and air temperature are collected from the Arduino-Uno while the rest of the input features are entered from the dataset [25] used in the experiment.

\section{B. Hardware Interface}

There are three components of the hardware interface: (1) LM35 temperature sensor are included in the Hardware Interface: LM35 is developed for indoor climate measurement; if the sensor and the Arduino is connected as in Fig. 2(a). The Arduino will begin receiving data from this sensor immediately; the Arduino-Uno board as shown in Fig. 2(b) by Arduino [21]. (2) The Arduino-Uno is a microcontroller board. The digital and analog input/output pins are given. This is coded in $\mathrm{C}$ language for every second from the temperature attached to the Arduino-Uno, for sending and receiving the temperature. The result shows that the present temperature affects the consumer at home. (3) HC05 Bluetooth: In order to start receiving android data through Bluetooth for this part as shown in Fig. 2(c). HC-05 only connects to android operating systems.

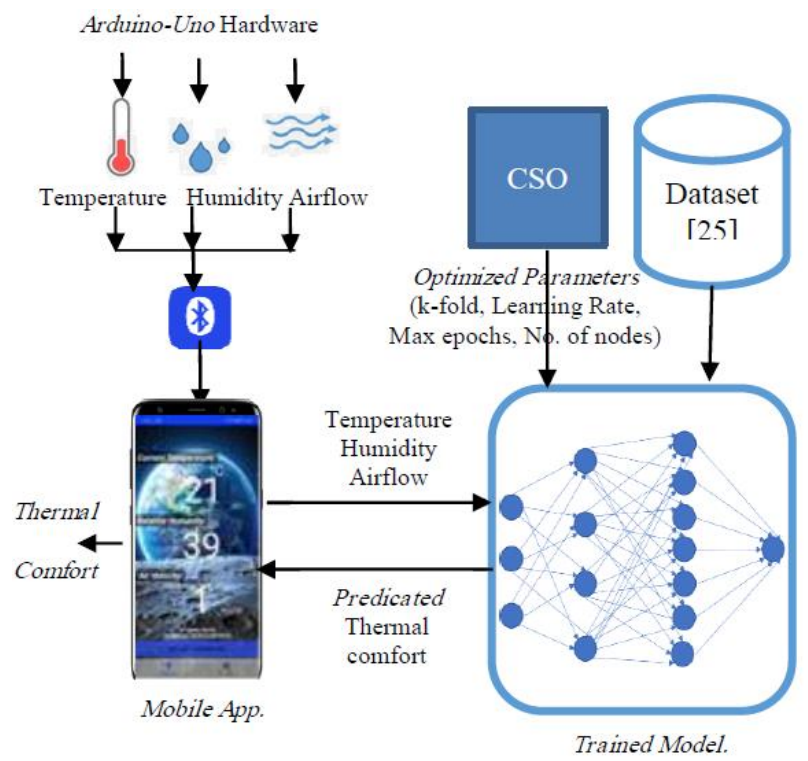

Fig. 1. Real-time Thermal Comfort Prediction Model.

TABLE I. INDEX LEVEL SCALES WITH THERMAL COMFORT

\begin{tabular}{|l|l|}
\hline Index Level & Thermal Comfort \\
\hline-2 & Cold \\
\hline-1 & Cool \\
\hline 0 & Comfort \\
\hline 1 & Slightly hot \\
\hline 2 & Hot \\
\hline
\end{tabular}




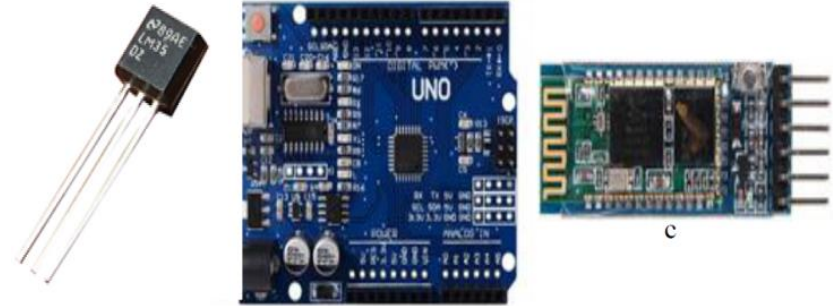

Fig. 2. Hardware Interface (a) LM35 Temperature Sensor, (b) Arduino-Uno and (c) Bluetooth HC-05.

\section{Mobile Application}

The smartphone framework is designed to obtain real-time temperatures from the hardware. Measured data will be transmitted privately via Bluetooth to the inhabitants' smartphones. Officers can still send input and aggregate information through a mobile network or WiFi to our backend server. Fig. 3(a) displays a range of standard mobile app user interfaces (UIs). The current temperature, air velocity, relative humidity and current thermal comfort/discomfort are provided to the operators.

The thermal navigation button bar leads the user to another big operation, where it consists of two predictions "Predicted temperature" and "Predicted thermal comfort/discomfort" as seen in Fig. 3(b). The user will be given an opportunity to alert the application to submit a default sound on the lock-screen for the expected temperature and thermal comfort/discomfort.
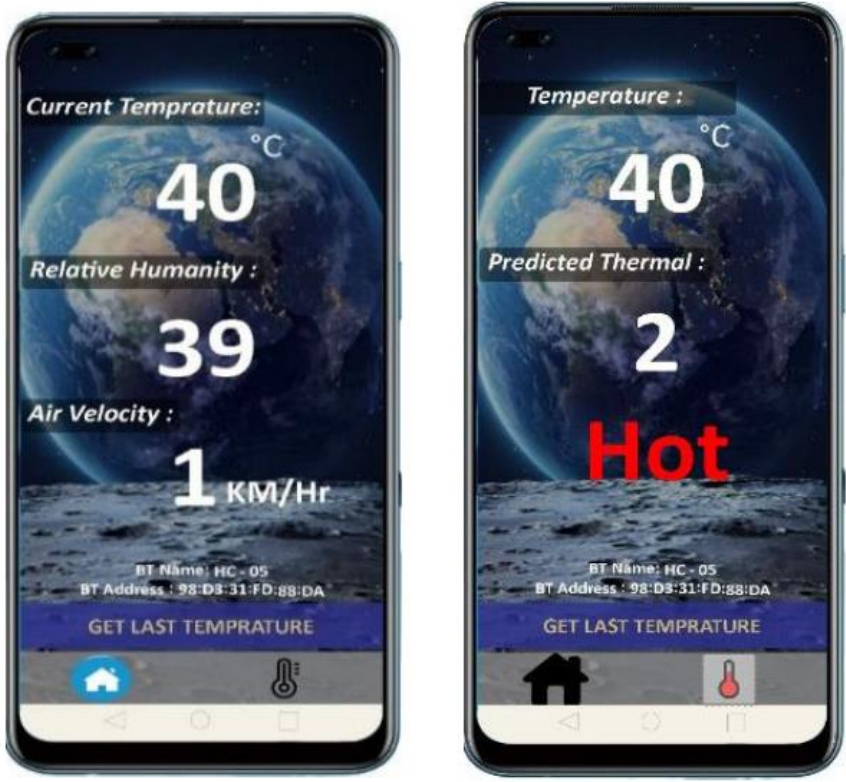

Fig. 3. (a) Home Page, (b) Thermal Page Interface.

\section{Proposed Methodology}

In this section, the approach proposed is clarified to efficiently solve problem of prediction of indoor thermal comfort using artificial neural network (ANN) with momentum function. The novel swarm algorithm (CSO) is utilized that automatically generate the most effective architecture model of NN to maximize classification performance and minimize the mean square error (MSE).

\section{A. Chicken Swarm Optimization (CSO)}

Chicken Swarm Optimization focused on computational optimization algorithms with bio-inspired behavior as discussed in [22]. There are a variety of communities in the chicken swarm. A dominant rooster, multiple hens and chicks are included each. The classification of these classes depends on the fitness values of the pigs. The best of the chickens would be roosters, each of whom would be the head rooster in a party. The most fitness-intensive chickens will be called chicks. The remainder is the hens. The hens chose the community in which they reside. The relationship of mother and child between hens and chicks is often formed randomly. In a collective there will be no shift in leadership, superiority and mother-and-child relationships. The hen selects classes arbitrarily in which to live and can only be changed for many generations. Chickens are hunting their group mate's rooster for food while avoiding consuming their own food, each group coordinates as a team and explore food according to a certain hierarchical order [23]. The chicken of the best fitness values of the next generation are picked of flocks.

$\mathrm{X}_{\mathrm{i}, \mathrm{j}}^{\mathrm{t}+1}=\mathrm{X}_{\mathrm{i}, \mathrm{j}}^{\mathrm{t}} *\left(1+\operatorname{Randn}\left(0, \sigma^{2}\right)\right)$

$\sigma^{2}=\left\{\begin{array}{c}1, \text { if } f_{i} \leq f_{k} \\ \exp \left(\frac{f_{k}-f_{i}}{\left|f_{i}\right|+\varepsilon}\right), \text { otherwise }\end{array} k \in[1, N], k \neq i\right.$

$X_{i, j}^{t+1}=X_{i, j}^{t}+S 1 *$ Rand $*\left(X_{r 1, j}^{t}-X_{i, j}^{t}\right)+S 2 *$ Rand $*$

$\left(\mathrm{X}_{\mathrm{r} 2, \mathrm{j}}^{\mathrm{t}}-\mathrm{X}_{\mathrm{i}, \mathrm{j}}^{\mathrm{t}}\right)$

$\left.S 1=\exp \left(\left(f_{i}-f_{r 1}\right) / a b s\left(f_{i}\right)+\varepsilon\right)\right)$

$S 2=\exp \left(\left(f_{r 2}-f_{i}\right)\right)$

$X_{i, j}^{t+1}=X_{i, j}^{t}+F L *\left(X_{m, j}^{t}-X_{i, j}^{t}\right)$

At time $t$, The $N$ number of chickens, are referred as $\mathrm{X}_{\mathrm{i}, \mathrm{j}}^{\mathrm{t}+1}$, where $i \in[1,2, \ldots, N], j \in[1,2, \ldots, D]$ in $D$-dimensional space. The optimization problem is actually the problem of finding the minimum value of nonlinear equations. Therefore, the best Par corresponds to the minimum fitness value. Fit, is the corresponding fitness value. Algorithm 1 defines the original CSO algorithm [23]. 


\section{Original Chicken Swarm Optimization (CSO) Algorithm} [21]

Step 1: Initialize a Maximal generations (M), Population size(pop), Dimension(d), How the chicken swarm can often be updated(G). The roosters population size (rPercent), hens accounts (hPercent), mother hens accounts for (mPercent).

Step 2: Initialize randomly using Gaussian random generator the size of (rooster rNum, hens hNum, chicks cNum, mother hens mNum).

Step 3: Evaluate the $\mathrm{N}$ chickens' fitness values, $\mathrm{t}=0$;

Step 4: Check If $(\mathrm{t} \% \mathrm{G}==0)$ then ort the chickens' fitness values and establish a swarm hierarchal order;

Divide different swarm groups, and identify the relationship between chicks' hens in a group

Step 5: For $\mathrm{i}=1: \mathrm{N}$ : Update roosters solutions, hen and chicken solutions (locations)

Check if $i==$ rooster thus, modify its rooster's location using Equation 1

Check if $i==$ hen thus, modify its hen's location using Equation 3

Check if $i==$ chick thus, update its chick's location using Equation 6

New solution evaluation;

Cheek if the new solution is better than the previous solution, then update it;

Check if $(t<M)$ go to step 4 else output results

\section{B. Learning Model: Momentum Back Propagation (MBP) Algorithm}

The dynamic back propagation (BP) approach was also used to adapt artificial neural networks to different problem typing patterns. One significant drawback of this system, however, is that it is highly dependent on these choices of momentum and size values [24]. Supervised learning will be needed for this study. In the classification, the momentum algorithm was used to find common properties from different classes. Also, it helps in enhancing the training speed and accuracy of finding values for weights so that given input and the computed output values are closely correctly match the known. Momentum also, consists of 3 phases. The first phase is the forward phase in which we begin the net and s(net) computation. The backward phase is the second phase that measures the error. The final and third phase is the weight update in which weights are updated from the output to the hidden layer and then to the input layers, if the error square is greater than the mean square error to get the final weights.

$s(n e t)=\frac{1}{1}+e^{-n e t}$

The backward phase calculates errors at all nodes using equations 1, 2.

For output error: $y_{1}\left(1-y_{1}\right) *\left(d-y_{1}\right)$

For hidden error:

$z_{1} *\left(1-z_{1}\right) * \sum_{k=1}^{m} s_{1} k w_{1} k$

The last step, if the Error square is greater than the MSE, thus it is going to apply weights update to get the final weights resulted using Equation 4.

$W_{\text {new }}=\mu * S * Z+\left[\alpha * W_{\text {old }}\right]$

After doing all of this, the final weights are used_for thermal comfort prediction in android application.

\section{EXPERIMENTS AND RESULTS}

\section{A. Dataset}

From July 2005 to July 2019 data are collected for one year, from weather archive in Cairo, Egypt [25]. Totally, there are 30,354 records in the dataset, and ten input features which are; time, air velocity, air temperature, global temperature, weight, height, sex, solar radiation, temperature gradient, relative humidity, and the eleven attribute is the index value that corresponds to the comfort value associated with the input features.

\section{B. Experiments Evaluation and Results}

The experiment results are shown based on the iterative nature of chicken swarm optimization algorithm. Table II shows some examples of predicted results of CSO.

Table II and Fig. 4 illustrate that optimal solution has been found by chicken swarm optimization algorithm where the objective value is to minimize the error. The performance comparison between the fine-tuned neural network experiments and optimization of neural network is using the chicken swarm optimization experiment (CSO-NN). We will note that CSO algorithm produces a little time. Therefore, a low overall complexity is for modelling and forecasting. The runtime can be reduced by 1.281 . It then enhances the credibility of the forecast and minimizes all MSE. The MBP architecture in Fig. 5 achieved a classification performance of $98,25 \%$ by training the classifier using 7 -folds.

Table III shows the overall confusion matrix that evaluated the performance of the developed model using the best architecture of MBA. The confusion matrix represents the five thermal comfort classes \{Cold, Cool, Comfort, Slightly hot, Hot $\}$. The results proved that the model developed is able to predict thermal comfort.

TABLE II. EXPERIMENTS RESULTS

\begin{tabular}{|l|l|l|l|l|}
\hline k-fold & $\begin{array}{l}\text { Learning } \\
\text { Rate }\end{array}$ & $\begin{array}{l}\text { Max } \\
\text { epochs }\end{array}$ & No. of nodes & Accuracy (\%) \\
\hline 27 & 0.2 & 2 & $(10,8,9,5)$ & 69.246 \\
\hline 17 & 0.4 & 44 & $(10,8,10,5)$ & 88.231 \\
\hline 21 & 0.4 & 62 & $(10,4,9,5)$ & 89.300 \\
\hline 9 & 0.6 & 66 & $(10,8,1,5)$ & 93.710 \\
\hline 9 & 0.2 & 22 & $(10,2,4,5)$ & 92.130 \\
\hline 7 & 0.5 & 46 & $(10,7,7,5)$ & 98.252 \\
\hline
\end{tabular}




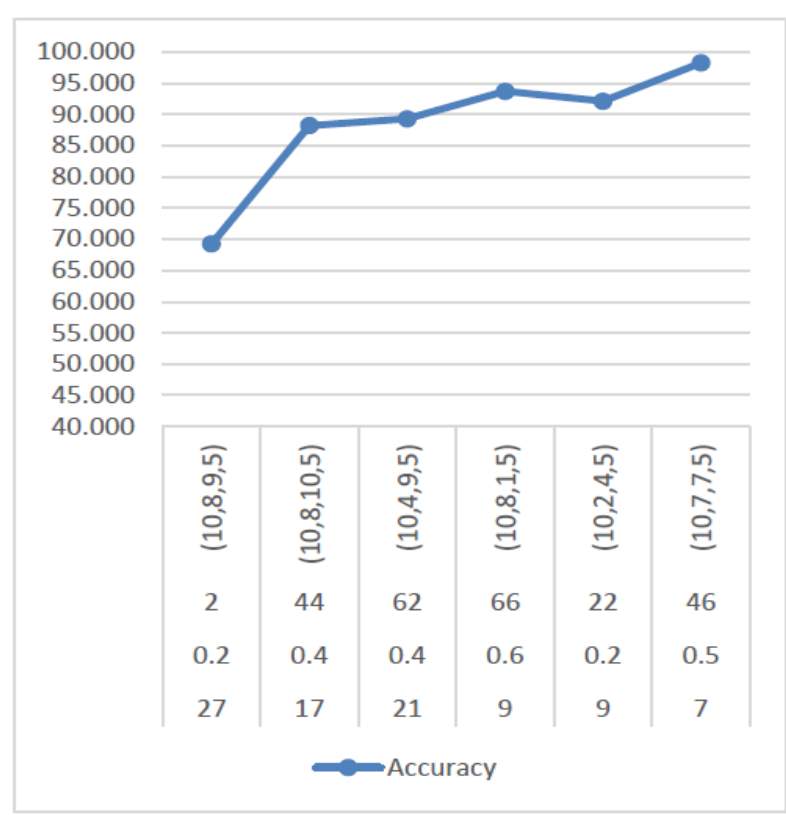

Fig. 4. Experiments Results of MBP.

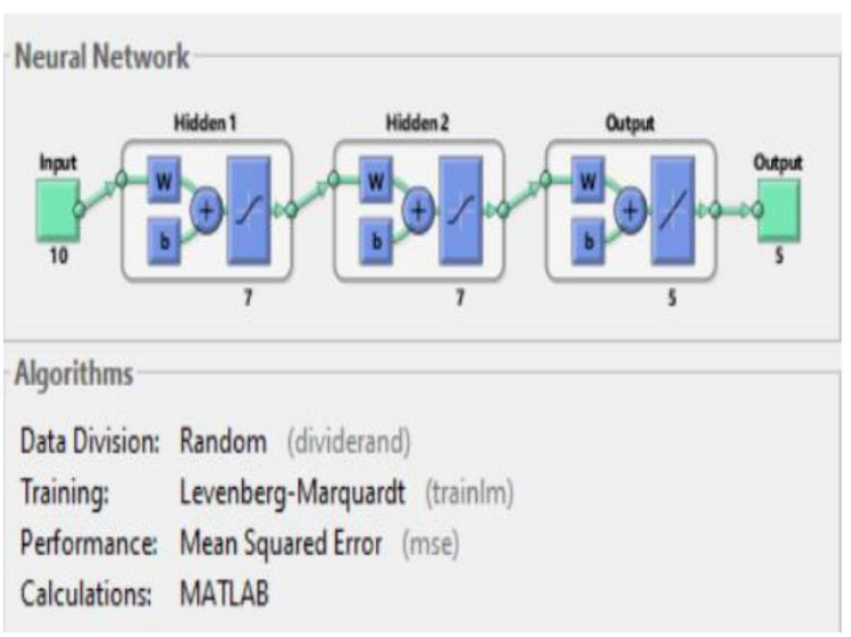

Fig. 5. Best Architecture of MBP using CSO.

TABLE III. CONFUSION MATRIX OF THERMAL ClASSES

\begin{tabular}{|l|l|l|l|l|l|}
\hline Class Label & Class 1 & Class 2 & Class 3 & Class 4 & Class 5 \\
\hline Class 1 (Cold) & 347 & 4 & 1 & 0 & 0 \\
\hline Class 2 (Cool) & 55 & 7536 & 57 & 4 & 0 \\
\hline Class 3 (Comfort) & 35 & 40 & 8120 & 69 & 0 \\
\hline Class 4 (Slightly hot) & 0 & 26 & 80 & 10820 & 82 \\
\hline Class 5 (Hot) & 0 & 0 & 18 & 60 & 3000 \\
\hline
\end{tabular}

\section{Evaluation Metrics}

As shown in Table IV, we calculate the evaluation metrics to the best structure of the trained neural network from CSO optimization that consists of 10 input features and 7 nodes in the first hidden layer, 7 nodes in the second hidden layer and 5 nodes in the output layer; with 46 epochs and 0.5 learning rate; we can see that the proposed method gave us a better result in all metrics.
TABLE IV. EVALUATION METRICS

\begin{tabular}{|l|l|l|l|}
\hline Metrics & $\begin{array}{l}\text { Results } \\
\mathbf{1 0 , 7 , 7 , 5}\end{array}$ & Formula & Evaluation Focus \\
\hline $\begin{array}{l}\text { Accuracy } \\
\text { acc) }\end{array}$ & 0.9825 & $\frac{t p+t n}{t p+f p+t n+f n}(11)$ & $\begin{array}{l}\text { Typically calculates the } \\
\text { percentage of accurate } \\
\text { forecasts over the total } \\
\text { number of measured } \\
\text { instances }\end{array}$ \\
\hline $\begin{array}{l}\text { Error Rate } \\
\text { (err) }\end{array}$ & 0.0175 & $\frac{f p+f n}{t p+f p+t n+f n}(12)$ & $\begin{array}{l}\text { The error of misclassification } \\
\text { tests the ratio of false } \\
\text { predictions to the actual } \\
\text { number of cases assessed }\end{array}$ \\
\hline $\begin{array}{l}\text { Sensitivity } \\
\text { (sn) }\end{array}$ & 0.9595 & $\frac{t p}{t p+f n}(13)$ & $\begin{array}{l}\text { Calculate accurately } \\
\text { classified fractions of positive } \\
\text { patterns }\end{array}$ \\
\hline $\begin{array}{l}\text { Specificity } \\
\text { (sp) }\end{array}$ & 0.9914 & $\frac{t n}{t n+f p}(14)$ & $\begin{array}{l}\text { Calculate the proportion of } \\
\text { the negatively patterns } \\
\text { classified correctly }\end{array}$ \\
\hline $\begin{array}{l}\text { Precision } \\
\text { (p) }\end{array}$ & 0.9774 & $\frac{t p}{t p+f p}(15)$ & $\begin{array}{l}\text { Determine the positive } \\
\text { patterns that are adequately } \\
\text { predicted in a positive class } \\
\text { by the total predicted patterns }\end{array}$ \\
\hline $\begin{array}{l}\text { F-Measure } \\
\text { (FM) }\end{array}$ & 0.9683 & $\frac{2 \times p \times r}{p+r}(16)$ & $\begin{array}{l}\text { Describes the harmony } \\
\text { among recall and precision } \\
\text { values }\end{array}$ \\
\hline $\begin{array}{l}\text { Negative } \\
\text { Predictive } \\
\text { Value }\end{array}$ & 0.9844 & $\frac{t n}{t n+f n}(17)$ & $\begin{array}{l}\text { The percentage of negative } \\
\text { test outcomes reported } \\
\text { correctly }\end{array}$ \\
\hline $\begin{array}{l}\text { False } \\
\text { Positive } \\
\text { Rate }\end{array}$ & 0.0086 & $\frac{f p}{f p+t n}(18)$ & $\begin{array}{l}\text { The risk that the null } \\
\text { hypothesis for the given test } \\
\text { will be denied falsely. }\end{array}$ \\
\hline $\begin{array}{l}\text { False } \\
\text { Discovery } \\
\text { Rate }\end{array}$ & 0.0226 & $\frac{f p}{f p+t p}(19)$ & $\begin{array}{l}\text { The significant features rate is } \\
\text { truly null }\end{array}$ \\
\hline & 0.0405 & $\frac{f n}{f n+t p}(20)$ & $\begin{array}{l}\text { Used to conceptualize Type I } \\
\text { error rates when evaluating } \\
\text { null hypotheses with many } \\
\text { comparisons. Intended to } \\
\text { monitor the estimated } \\
\text { percentage of false } \\
\text { discoveries. }\end{array}$ \\
\hline
\end{tabular}

\section{CONCLUSIONS}

Thermal comfort impacts working efficiency at work sites, and it is very important for consumers to be conscious of the performance of such thermal environments. This paper demonstrates the practicability of the intelligent thermal comfort application for individual's thermal comfort prediction in the android application by automatic collect the relative humidity, air temperature and air velocity. The predication model proposed is based on momentum algorithm which achieved an accuracy result of $98.25 \%$ for thermal comfort, taking the final weights of the classification model, which is optimized using bio-inspired optimization algorithm (CSO). Furthermore, the analysis of the results showed that our proposed optimization model provides the optimal solution that achieved the minimum MSE of 1.7477.

\section{REFERENCES}

[1] Y. Song, F. Mao and Q. Liu, "Human Comfort in Indoor Environment: A Review on Assessment Criteria, Data Collection and Data Analysis Methods," in IEEE Access, vol. 7, pp. 119774-119786, 2019, doi: 10.1109/ACCESS.2019.2937320. 
[2] P. K. Rosier, "Comfort theory and practice: A vision for holistic health care and research", Clin. Nurse Spec., vol. 19, pp. 49, Jan. 2005.

[3] K. C. Parsons, Human thermal environments: the effects of hot, moderate, and cold environments on human health, comfort, and performance. Boca Raton: CRC Press, 2014.

[4] N. Ma, D. Aviv, H. Guo, and W. W. Braham, "Measuring the right factors: A review of variables and models for thermal comfort and indoor air quality," Renewable and Sustainable Energy Reviews, vol. 135, p. 110436, 2021.

[5] Yao R, Li B, Liu J (2009). R. Yao, B. Li, and J. Liu, "A theoretical adaptive model of thermal comfort - Adaptive Predicted Mean\&nbsp;Vote\&nbsp;(aPMV)," Building and Environment, vol. 44, no. 10, pp. 2089-2096, 2009.

[6] D. Kong, H. Liu, Y. Wu, B. Li, S. Wei, and M. Yuan, "Effects of indoor humidity on building occupants' thermal comfort and evidence in terms of climate adaptation," Building and Environment, vol. 155, pp. 298307, 2019.

[7] H. Yan, Q. Liu, H. Zhang, H. Wang, H. Li, and L. Yang, "Difference in the thermal response of the occupants living in northern and southern China," Energy and Buildings, vol. 204, p. 109475, 2019.

[8] B. Li and R. Yao, "Building energy efficiency for sustainable development in China: challenges and opportunities," Building Research \&amp; Information, vol. 40, no. 4, pp. 417-431, 2012.

[9] X. Yuan, Y. Pan, J. Yang, W. Wang, and Z. Huang, "Study on the application of reinforcement learning in the operation optimization of HVAC system," Building Simulation, vol. 14, no. 1, pp. 75-87, 2020.

[10] M. Rocca, "Health and well-being in indoor work environments: a review of literature," 2017 IEEE International Conference on Environment and Electrical Engineering and 2017 IEEE Industrial and Commercial Power Systems Europe (EEEIC / I\&amp;CPS Europe), 2017.

[11] P. O. Fanger et al., "Thermal comfort. Analysis and applications in environmental engineering." Thermal comfort. Analysis and applications environmental engineering. 1970.

[12] P. Bluyssen, The indoor environment handbook: how to make buildings healthy and comfortable. London: Earthscan, 2015.

[13] S. Zhang, C. Zhu, J. K. O. Sin, and P. K. T. Mok, "A novel ultrathin elevated channel low-temperature poly-Si TFT," IEEE Electron Device Lett., vol. 20, pp. 569-571, Nov. 2007.

[14] Thermal environmental conditions for human occupancy. Atlanta, GA: ASHRAE, 2017. K. Chen, Y. Jiao, and E. S. Lee, “Fuzzy adaptive networks in thermal comfort," Appl. Math. Lett, vol. 19, no. 5, pp. 420426, 2006.

[15] D. Markov, "Practical evaluation of the thermal comfort parameters", Proc. Annu. Int. Course Ventilation Indoor Climate, pp. 158-170, Oct. 2002.

[16] T. Chaudhuri, D. Zhai, Y. C. Soh, H. Li, and L. Xie, "Random forest based thermal comfort prediction from gender-specific physiological parameters using wearable sensing technology," Energy and Buildings, vol. 166, pp. 391-406, 2018.

[17] N. Seydoux, K. Drira, N. Hernandez and T. Monteil, "Autonomy through knowledge: How IoT-o supports the management of a connected apartment", Proc. Semantic Web Technol. Internet Things (SWIT) Workshop ISWC CEUR WS, 2016.

[18] W. Zhang, W. Hu, and Y. Wen, "Thermal Comfort Modeling for Smart Buildings: A Fine-Grained Deep Learning Approach," IEEE Internet of Things Journal, vol. 6, no. 2, pp. 2540-2549, 2019.

[19] T. Chaudhuri, Y. C. Soh, H. Li, L. Xie, "Machine learning based prediction of thermal comfort in buildings of equatorial Singapore", IEEE International Conference on Smart Grid and Smart Cities (ICSGSC), pp. 72-77, July 2017.

[20] S. Atthajariyakul and T. Leephakpreeda, "Neural computing thermal comfort index for HVAC systems," Energy Conversion and Management, vol. 46, no. 15-16, pp. 2553-2565, 2005.

[21] “ArduinoBoardUno," Arduino. [Online]. Available: https://www. arduino.cc/en/pmwiki.php?n=Main\%2FarduinoBoardUno. [Accessed: 14-Feb-2021].

[22] A. Darwish, "Bio-inspired computing: Algorithms review, deep analysis, and the scope of applications," Future Computing and Informatics Journal, vol. 3, no. 2, pp. 231-246, 2018. mhttp://www.journals.elsevier. com/future-computing-and-informatics-journal/.

[23] Meng X., Liu Y., Gao X., Zhang H, "A New Bio-inspired Algorithm: Chicken Swarm Optimization”. In: Tan Y et al., Coello C.A.C. (eds) Advances in Swarm Intelligence. ICSI, Part I, LNCS 8794, pp. 86-94, 2014.

[24] Chien-Cheng Yu and Bin-Da Liu, "A backpropagation algorithm with adaptive learning rate and momentum coefficient," Proceedings of the 2002 International Joint Conference on Neural Networks. IJCNN'02 (Cat. No.02CH37290).

[25] Weather in 243 countries of the world, 14-Feb-2021. [Online]. Available: https://rp5.ru/. [Accessed: 14-Feb-2021]. 